\title{
Incidence, risk factors, and prognosis in patients with primary hepatocellular carcinoma and lung metastasis: a population-based study
}

This article was published in the following Dove Medical Press journal: Cancer Management and Research

\section{Chao Wu \\ Xudong Ren \\ Quanbao Zhang}

Department of General Surgery, Huashan Hospital, Fudan University, Shanghai 200040, China
Correspondence: Quanbao Zhang Department of General Surgery, Huashan Hospital, Fudan University, 12 Urumqi Road (M), Shanghai 200040, China

Tel +86 2I 52887174

$\mathrm{Fax}+862152887174$

Email zhangquanbao1979@hotmail.com
Aims: The study aims to explore the incidence, risk factors, and prognosis in patients with primary hepatocellular carcinoma (HCC) and synchronous lung metastasis using a large-scale population-based cancer registry database.

Patients and methods: Data of 33,177 HCC patients were retrieved from the Surveillance, Epidemiology, and End Results (SEER) database from 2010 to 2015. Multivariate logistic and Cox regression model analysis were applied for the recognition of risk factors and prognostic factors associated with lung metastasis among HCC patients. The overall survival and cancerspecific survival of HCC patients with initial pulmonary metastasis were estimated by KaplanMeier analysis, and the survival curves were compared by log-rank tests.

Results: Total 2,084 (6.28\%) HCC patients diagnosed with initial pulmonary metastasis were enrolled for analysis. Male gender, younger age, non-white race, unmarried status, uninsured status, elevated alpha-fetoprotein, larger primary liver tumor size, positive lymph node status, synchronal bone or brain metastasis, and tumor poor pathological differentiation were relevant to higher risk of lung metastasis in HCC cohort. The 1-, 3-, 5-year overall survival and cancerspecific survival rates for HCC lung metastasis patients were $12.8 \%$ vs $15.3 \%, 4.0 \%$ vs $5.7 \%$, and $1.6 \%$ versus $2.4 \%$, respectively. The median overall and cancer-specific survival time in HCC lung metastasis group were both 3 months, while the corresponding time in HCC lung metastasis-free group were 19 and 25 months $(P<0.05)$. Older age, unmarried status, poor tumor differential grade, and absence of surgery were identified as unfavorable prognosis factors.

Conclusion: The survival of patients with HCC lung metastasis was dismal. Several clinicopathological factors were found to be significantly relevant to the development and prognosis of HCC lung metastasis. These new findings could be useful for a precise and individualized therapeutic schedule.

Keywords: liver cancer, lung metastasis, risk factor, prognosis factor, SEER

\section{Introduction}

Hepatocellular carcinoma (HCC) is the most frequent subtype of primary liver cancer with high lethality. ${ }^{1}$ Recent statistical data demonstrated that the 5-year survival rate of HCC is still below $20 \%{ }^{2,3}$ One of reasons responsible for high mortality is the extrahepatic spread of primary tumor loci with partly elucidated mechanism. ${ }^{4,5}$ Till now, lung is considered as the most favored organ for HCC metastatic colonization, counting for $51 \%$ of all extrahepatic metastasis. ${ }^{6}$ Result from a large-scale population study showed that the 1-year overall survival (OS) and cancer-specific survival (CSS) was $10 \%$ and $12.6 \%$ in patients diagnosed with HCC lung metastasis (HCCLM). ${ }^{7}$ 
Previously, HCC initiated with lung metastasis was regarded as the late stage of disease with poor survival. ${ }^{1}$ With the conceptual update in treatment modalities, new attempts, such as simultaneous hepatectomy and pulmonary metastasectomy, have been made by surgeons in selected individuals to achieve a better survival. ${ }^{8-10}$ In addition, Yang et al elucidated that patients receiving combined treatment had a better survival by analyzing 76 consecutive HCC patients initially presenting with lung metastasis. ${ }^{11}$ However, primary and secondary prevention strategies also played a pivotal role in cancer remedy. Early recognition of patients with high risk for development of HCCLM and subsequent surveillance should be proposed. A previous article illustrated that HCC $<7 \mathrm{~cm}$ in diameter had higher risk of forming lung metastasis after primary liver cancer resection. ${ }^{12}$ Due to a low prevalence of HCCLM, a large-scale population-based study is needed for systematic identification of the morbidity, potential risk factors, and clinical prognosis of initial HCCLM patients.

In the present research, we retrospectively reviewed data from the Surveillance, Epidemiology, and End Results (SEER) database to explore the actual incidence and identify factors contributing to initially diagnosed HCCLM. Furthermore, stratified survival estimates and variables affecting either OS or CSS recognition were also conducted.

\section{Patients and methods}

\section{Data source and patient enrollment}

Original data were retrieved from the SEER database maintained by the National Cancer Institute. The SEER program consists of 18 population-based cancer registries, covering $\sim 28 \%$ of all population in the USA. The distant metastasis status were collected until 2010 due to the intrinsic reason of the database and the latest revision was released on April 16,2018 . The inclusion criteria were as follows: 1) primary site labeled as "C22.0 Liver" and ICD-O-3 histology/behavior marked with "8,170/3: hepatocellular carcinoma, not otherwise specified," "8,171/3: hepatocellular carcinoma, fibrolamellar," "8,172/3: hepatocellular carcinoma, scirrhous," "8,173/3: hepatocellular carcinoma, spindle cell variant," "8,174/3: hepatocellular carcinoma, clear cell type," or " $8,175 / 3$ : hepatocellular carcinoma, pleomorphic type"; 2) the year at diagnosis from 2010 to 2015; and 3) the age at diagnosis older than 18 years. Individuals who had unclear TNM stage record, unknown survival time, missing cause of death, or unknown diagnostic confirmation were subsequently excluded. For further analysis, cases were grouped by the existence of lung metastasis (Figure 1).

We obtained the SEER data access permission before the project initiation. The study complied with the Declaration

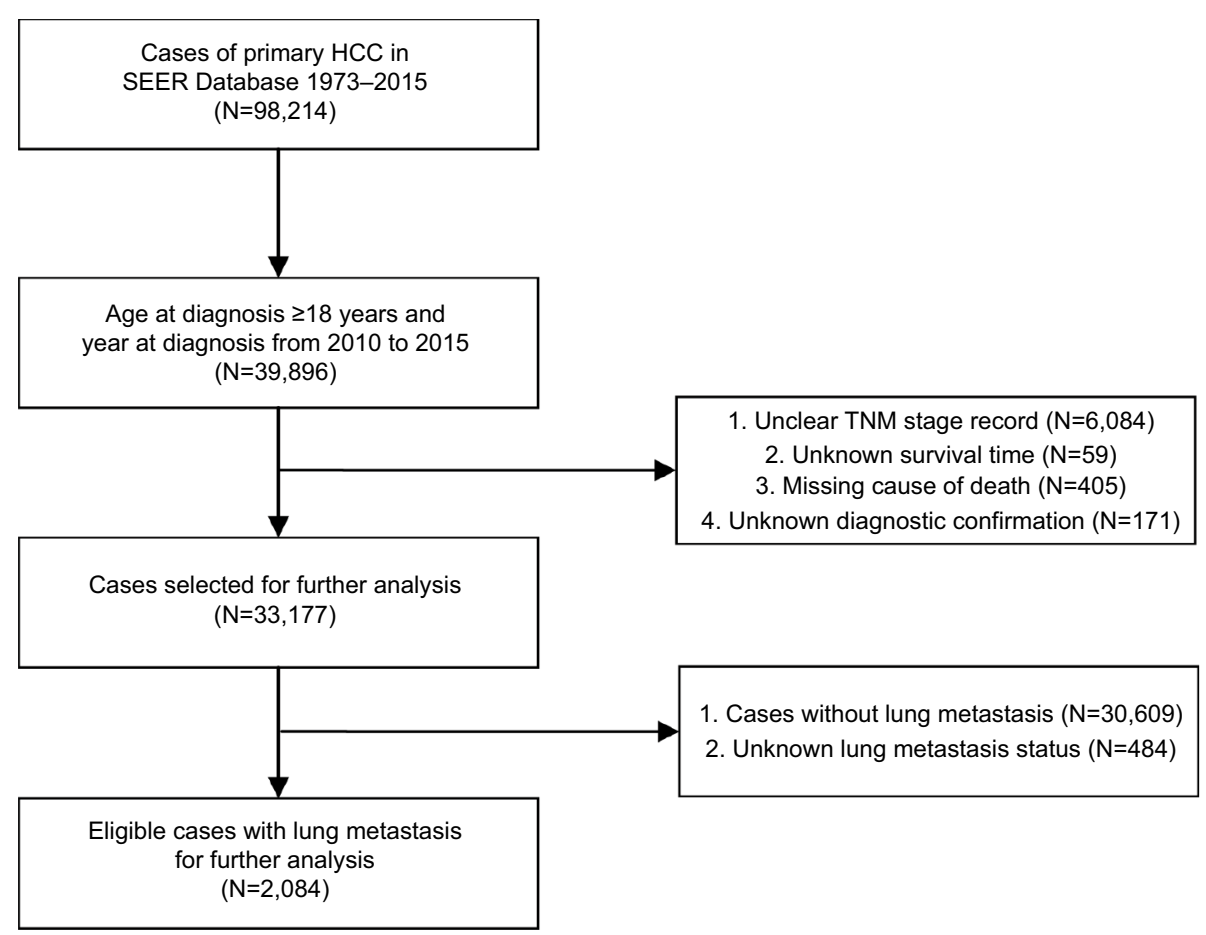

Figure I Flowchart of the enrolled patients in the study according to inclusion and exclusion criterion. Abbreviations: HCC, hepatocellular carcinoma; SEER, Surveillance, Epidemiology, and End Results. 
of Helsinki and followed the ethical principles of Huashan Hospital, Fudan University.

\section{Variables definition and stratification}

The definition of incidence was the proportion of patients with lung metastasis in the entire HCC patients. We stratified total cohort by age at diagnosis, gender, race, marital status at diagnosis, insurance status, alpha-fetoprotein (AFP) interpretation, maximum of tumor size, 7th AJCC TNM staging, lymph node status, surgery, pathological grade, and other distant site metastasis (brain and bone). Liver metastasis and pathological subtypes were not included for analysis because the records were unable to be distinguished clearly. The variable race comprised of white, black, and others (American Indian/Alaska Native, Asian/Pacific Islander). AFP level was interpreted as elevated, normal, borderline, as well as unknown. Surgery for primary site was classified as no surgery, local tumor destruction, surgery, and unknown. OS was defined as the duration between the surgery and death or the last follow-up, while the CSS was the period between the surgery and the death due to cancer.

\section{Statistical analyses}

Data were downloaded by SEER*Stat Software version 8.3.5 (National Cancer Institute, Bethesda, MD, USA). SPSS version 13.0 (IBM Inc., Chicago, IL, USA) was used for statistical analysis, and survival curves were generated using GraphPad Prism version 6.0 (GraphPad-Prism Software Inc., San Diego, $\mathrm{CA}, \mathrm{USA})$. Continuous variables were presented as mean $\pm \mathrm{SD}$ or median (minimum, maximum), and the categorical variables were shown as number (percent). Association between categorical data was analyzed using the chi-squared test or Fisher's exact test, while continuous values using Student's $t$-test or Mann-Whitney test, when appropriate. The KaplanMeier method and Cox regression analysis were applied to determine prognostic factors associated with OS or CSS. Two tailed $P<0.05$ was regarded as statistically significant.

\section{Results}

\section{Incidence of HCCLM}

Among finally enrolled 33,177 patients with HCC from 2010 to $2015,2,084$ patients were initially diagnosed with HCCLM with an incidence rate $6.28 \%$ (Figure 1). No statistical significance could be found between different years. Only 44 (2.1\%) individuals with HCCLM received surgery (including local tumor destruction), while remaining $97.8 \%$ patients treated conservatively (Table 1 ).

\section{Risk factors for HCCLM}

As depicted in Table 1, male gender (OR: 1.131, 95\% CI: 1.014-1.260, $P=0.026$ ), non-white race, unmarried status (OR: $1.313,95 \%$ CI: $1.186-1.454, P<0.001$ ), uninsured status (OR: 1.988, 95\% CI: 1.661-2.380, $P<0.001$ ), maximum primary liver tumor size over $50 \mathrm{~mL}(\mathrm{~mm})$, positive lymph node status (OR: 4.326, 95\% CI: 3.838-4.875, $P<0.001$ ), synchronal bone (OR: $5.495,95 \%$ CI: 4.827-6.255, $P<0.001$ ) or brain metastasis (OR: 11.492, 95\% CI: 7.892-16.734, $P<0.001$ ), and tumor poor pathological differentiation were relevant to higher risk of initial HCCLM. Additionally, patients with HCC older than 40 years or normal AFP level had lower risk of developing lung metastasis (Table 1).

\section{Survival and prognostic factors of HCCLM patients}

Median OS in HCC lung metastasis-free (HCCLMF) group and HCCLM group were 19 months and 3 months, while the median CSS in the group of HCCLMF and HCCLM were 25 months and 3 months. The 1-, 3-, and 5-year OS rates for HCC patients without or with lung metastasis were $59.8 \%$ vs $12.8 \%, 33.4 \%$ vs $4.0 \%$, and $24.1 \%$ versus $1.6 \%$, respectively (Figure 2A). In addition, the homologous proportions of CSS were $65.2 \%$ vs $15.3 \%, 41.1 \%$ vs $5.7 \%$, and $32.4 \%$ versus $2.4 \%$ (Figure 2B). Further, log-rank analysis revealed that age at diagnosis, gender, lymph node status, maximum primary tumor size, tumor differentiation, AFP level interpretation, and surgical intervention had impact on HCCLM cohort patients' OS with statistical significance $(P<0.05$, Figure 3$)$. Besides factors above, bone metastasis was also associated with CSS in HCCLM cohort $(P<0.05$, Figure 4$)$.

Using the univariate and multivariate Cox regression model, age at diagnosis older than 40 years, unmarried status (HR: 1.134, 95\% CI: 1.019-1.261, $P=0.021$ ), poor differentiation (HR: $1.732,95 \% \mathrm{CI}: 1.355-2.213, P<0.001$ ) or undifferentiation (HR: 1.732, 95\% CI: 1.355-2.213, $P<0.001$ ), and surgical intervention (including local tumor destruction and surgery) were identified as worse prognosis factors for OS (Table 2). Similarly, these factors were also associated with a higher risk of mortality caused by cancer (Table 3 ).

\section{Discussion}

HCCLM is a Gordian knot for clinicians because of its poor survival and limited effective treatment modalities. Previous articles mainly focused on the lung metastasis followed by primary liver tumor resection, while little is known about the simultaneous HCC and lung metastatic tumor. In the current 
Table I Baseline clinical demographics and multivariable logistic regression for analyzing risk factor for initial lung metastasis of primary hepatocellular carcinoma patients in SEER database (2010-20I5)

\begin{tabular}{|c|c|c|c|c|c|c|}
\hline \multirow[t]{2}{*}{ Variables } & \multicolumn{2}{|c|}{ Lung metastasis free } & \multicolumn{2}{|c|}{ Lung metastasis } & \multirow[t]{2}{*}{ OR $(95 \% \mathrm{Cl})$} & \multirow[t]{2}{*}{$P$-value } \\
\hline & \begin{tabular}{|l}
$\begin{array}{l}\text { No. of } \\
\text { patients }\end{array}$ \\
\end{tabular} & $\%$ & $\begin{array}{l}\text { No. of } \\
\text { patients }\end{array}$ & $\%$ & & \\
\hline Gender & & & & & & 0.026 \\
\hline Female & 7,111 & 23.2 & 440 & 21.1 & I (Reference) & $\mathrm{I}$ \\
\hline Male & 23,498 & 76.8 & $\mathrm{I}, 644$ & 78.9 & $1.131(1.014-1.260)$ & 0.026 \\
\hline Age at diagnosis (years) & & & & & & $<0.001$ \\
\hline $18-39$ & 298 & 1.0 & 61 & 2.9 & I (Reference) & $\mathrm{I}$ \\
\hline $40-59$ & 10,484 & 34.2 & 758 & 36.4 & $0.353(0.266-0.470)$ & $<0.001$ \\
\hline $60-79$ & 16,992 & 55.5 & $I, 05 \mid$ & 50.4 & $0.302(0.228-0.40 \mathrm{I})$ & $<0.001$ \\
\hline$\geq 80$ & 2,835 & 9.3 & 214 & 10.3 & $0.369(0.27 I-0.502)$ & $<0.001$ \\
\hline Race & & & & & & $<0.001$ \\
\hline White & 21,304 & 69.6 & $\mathrm{I}, 322$ & 63.4 & \begin{tabular}{|l} 
I (Reference) \\
\end{tabular} & $\mathrm{I}$ \\
\hline Black & 4,192 & 13.7 & 361 & 17.3 & $1.388(1.230-1.566)$ & $<0.001$ \\
\hline Others & 4,960 & 16.2 & 392 & 18.8 & $1.274(1.133-1.432)$ & $<0.001$ \\
\hline Unknown & 153 & 0.5 & 9 & 0.5 & NA & NA \\
\hline Marital status at diagnosis & & & & & & $<0.001$ \\
\hline Married & 22,396 & 73.2 & 1,429 & 68.6 & I (Reference) & $\mathrm{I}$ \\
\hline Unmarried & 6,602 & 21.5 & 553 & 26.5 & $1.313(1.186-1.454)$ & $<0.001$ \\
\hline Unknown & $1,6 \mathrm{II}$ & 5.3 & 102 & 4.9 & NA & NA \\
\hline Insurance status & & & & & & $<0.001$ \\
\hline Insured & 29,010 & 94.8 & $\mathrm{I}, 893$ & 90.8 & I (Reference) & $\mathrm{I}$ \\
\hline Uninsured & 1,110 & 3.6 & 144 & 6.9 & $1.988(1.66 \mathrm{I}-2.380)$ & $<0.001$ \\
\hline Unknown & 489 & 1.6 & 47 & 2.3 & NA & NA \\
\hline Year at diagnosis & & & & & & 0.968 \\
\hline 2010 & 4,374 & 14.3 & 295 & 14.2 & I (Reference) & $\mathrm{I}$ \\
\hline 2011 & 4,655 & 15.2 & 324 & 15.6 & $1.032(0.877-1.215)$ & 0.705 \\
\hline 2012 & 5,034 & 16.5 & 355 & 17.0 & $1.046(0.89|-| .227)$ & 0.584 \\
\hline 2013 & 5,296 & 17.3 & 361 & 17.3 & 1.011 (0.862-1.185) & 0.896 \\
\hline 2014 & 5,576 & 18.2 & 371 & 17.8 & $0.987(0.842-1.155)$ & 0.866 \\
\hline 2015 & 5,674 & 18.5 & 378 & 18.1 & $0.988(0.844-1.156)$ & 0.878 \\
\hline Alpha-fetoprotein interpretation & & & & & & $<0.001$ \\
\hline Positive/elevated & 17,845 & 58.3 & $\mathrm{I}, 4 \mathrm{I}$ & 67.9 & I (Reference) & $\mathrm{I}$ \\
\hline Negative/normal & 7,062 & 23.1 & 211 & 10.1 & $0.377(0.325-0.437)$ & $<0.001$ \\
\hline Borderline & 65 & 0.2 & 6 & 0.3 & $1.165(0.504-2.693)$ & 0.721 \\
\hline Unknown & 5,637 & 18.4 & 453 & 21.7 & NA & NA \\
\hline Maximum primary tumor size $(\mathrm{mm})$ & & & & & & $<0.001$ \\
\hline $0-20$ & 3,364 & 11.0 & 69 & 3.3 & I (Reference) & $\mathrm{I}$ \\
\hline $20-50$ & 13,101 & 42.8 & 234 & 11.2 & $0.87 \mid(0.664-|| 42)$. & 0.317 \\
\hline $50-100$ & \begin{tabular}{|l|l|}
7,737 \\
\end{tabular} & 25.3 & 541 & 26.0 & $3.409(2.645-4.394)$ & $<0.001$ \\
\hline$\geq 100$ & 3,970 & 13.0 & 605 & 29.0 & $7.430(5.768-9.57 \mathrm{I})$ & $<0.001$ \\
\hline Unknown & 2,437 & 7.9 & 635 & 30.5 & NA & NA \\
\hline 7th AJCC TNM staging & & & & & & $<0.001$ \\
\hline $\mathrm{I}$ & 13,735 & 44.9 & 0 & 0.0 & I (Reference) & $\mathrm{I}$ \\
\hline II & 6,615 & 21.6 & 0 & 0.0 & NA & NA \\
\hline III & 6,078 & 19.9 & 0 & 0.0 & NA & NA \\
\hline IV & 4,181 & 13.6 & 2084 & 100 & $1.498(1.472-1.525)$ & $<0.001$ \\
\hline Lymph node status & & & & & & $<0.001$ \\
\hline Negative & 28,144 & 91.9 & 1,290 & 61.9 & I (Reference) & $\mathrm{I}$ \\
\hline Positive & 2,073 & 6.8 & 411 & 19.7 & $4.326(3.838-4.875)$ & $<0.001$ \\
\hline Unknown & 392 & 1.3 & 383 & 18.4 & NA & NA \\
\hline Bone metastasis & & & & & & $<0.001$ \\
\hline No & 29,436 & 96.2 & $\mathrm{I}, 667$ & 80.0 & I (Reference) & $\mathrm{I}$ \\
\hline Yes & 1,128 & 3.7 & 351 & 16.8 & $5.495(4.827-6.255)$ & $<0.001$ \\
\hline Unknown & 45 & 0.1 & 66 & 3.2 & NA & NA \\
\hline
\end{tabular}


Table I (Continued)

\begin{tabular}{|c|c|c|c|c|c|c|}
\hline \multirow[t]{2}{*}{ Variables } & \multicolumn{2}{|c|}{ Lung metastasis free } & \multicolumn{2}{|c|}{ Lung metastasis } & \multirow[t]{2}{*}{ OR $(95 \% \mathrm{Cl})$} & \multirow[t]{2}{*}{$P$-value } \\
\hline & $\begin{array}{l}\text { No. of } \\
\text { patients }\end{array}$ & $\%$ & $\begin{array}{l}\text { No. of } \\
\text { patients }\end{array}$ & $\%$ & & \\
\hline Brain metastasis & & & & & & $<0.001$ \\
\hline No & 30,501 & 99.7 & 1960 & 94.1 & I (Reference) & $\mathrm{I}$ \\
\hline Yes & 65 & 0.2 & 48 & 2.3 & $11.492(7.892-16.734)$ & $<0.001$ \\
\hline Unknown & 43 & 0.1 & 76 & 3.6 & NA & NA \\
\hline Surgery for primary site & & & & & & $<0.001$ \\
\hline No surgery & 22,213 & 72.6 & 2038 & 97.8 & I (Reference) & $\mathrm{I}$ \\
\hline Local tumor destruction ${ }^{\mathrm{a}}$ & 3,613 & 11.8 & 19 & 0.9 & $0.057(0.036-0.090)$ & $<0.001$ \\
\hline Surgery & 4,712 & 15.4 & 25 & 1.2 & $0.058(0.039-0.086)$ & $<0.001$ \\
\hline Unknown & 71 & 0.2 & 2 & 0.1 & NA & NA \\
\hline Grade & & & & & & $<0.001$ \\
\hline Well differentiated & 3,424 & 11.2 & 103 & 4.9 & I (Reference) & 1 \\
\hline Moderately differentiated & 5,225 & 17.1 & 236 & 11.3 & $1.50 \mathrm{I}(1.187-1.900)$ & 0.001 \\
\hline Poorly differentiated & 2,140 & 7.0 & 276 & 13.3 & $4.287(3.397-5.410)$ & $<0.001$ \\
\hline Undifferentiated & 167 & 0.5 & 21 & 1.0 & $4.180(2.550-6.853)$ & $<0.001$ \\
\hline Unknown & 19,653 & 64.2 & $\mathrm{I}, 448$ & 69.5 & NA & NA \\
\hline
\end{tabular}

Note: ${ }^{2}$ Local tumor destruction includes percutaneous ethanol injection, heat-radiofrequency ablation, cryosurgery, photodynamic therapy, electrocautery, fulguration (includes use of hot forceps for tumor destruction), laser, and others (ultrasound, acetic acid).

Abbreviation: SEER, Surveillance, Epidemiology, and End Results.

A

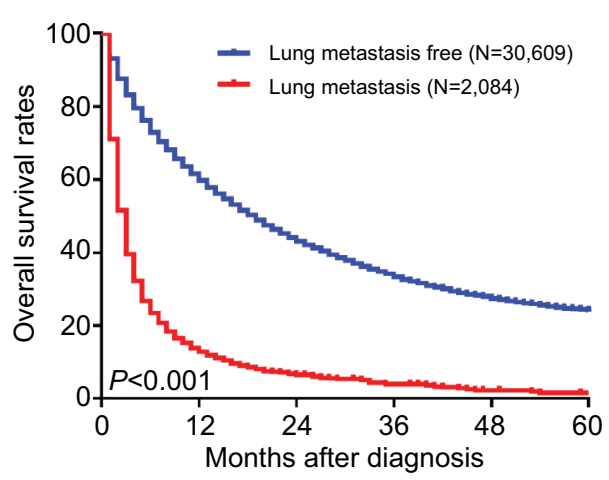

Number of subjects at risk

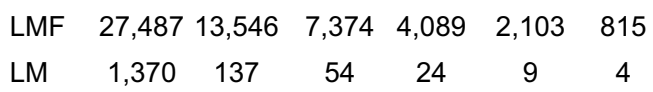

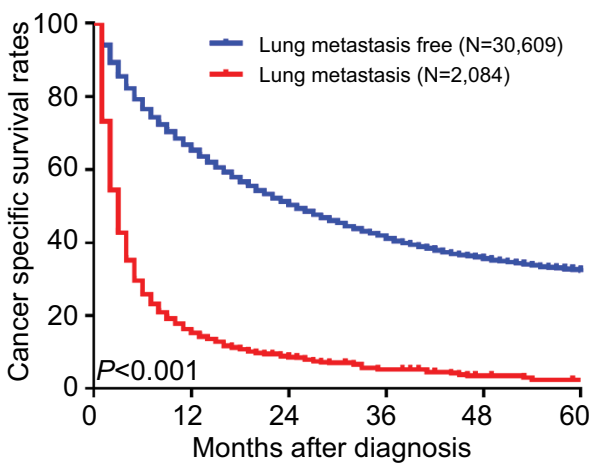

Number of subjects at risk

$\begin{array}{lcccccc}\text { LMF } & 27,487 & 13,546 & 7,374 & 4,089 & 2,103 & 815 \\ \text { LM } & 1,370 & 137 & 54 & 24 & 9 & 4\end{array}$

Figure 2 Kaplan-Meier analysis of (A) overall survival and (B) cancer-specific survival in hepatocellular carcinoma patients with or without initial lung metastasis. Abbreviations: LM, lung metastasis; LMF, lung metastasis free.

study, the epidemiological result indicated that $6.28 \%$ patients with HCC presented with synchronous lung metastasis. Similarly, result from a Chinese cohort revealed that 39 of 862 (4.5\%) HCC patients presented with initial lung metastasis. ${ }^{13}$ However, a national follow-up survey of primary liver cancer in Japan showed that more than two-fifths of HCC patients developed pulmonary metastatic tumor. ${ }^{14}$ The difference between our result and results from Japan study may be due to the discrepancy of enrolled patients. Our study only focused on the initial HCCLM cases, while Japan group analyzed all pulmonary metastatic cases during HCC progression. But, our result could be underestimated on account of the fact that asymptomatic HCCLM patients are unable to be detected. Strikingly, application of newly effective treatment modalities by clinicians may contribute to a slight increase in OS and CSS compared with data 3 years ago. ${ }^{7}$

Follow-up analysis identified several risk factors of HCCLM, including younger patient ( $<40$ years), non-white race, unmarried or uninsured status, elevated AFP level, larger primary tumor size, positive lymph node status, syn- 


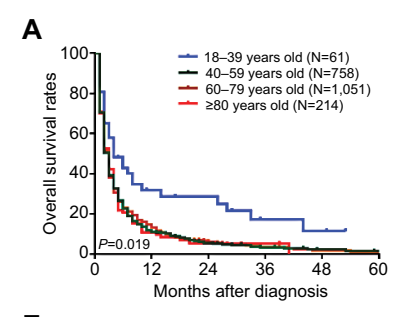

E
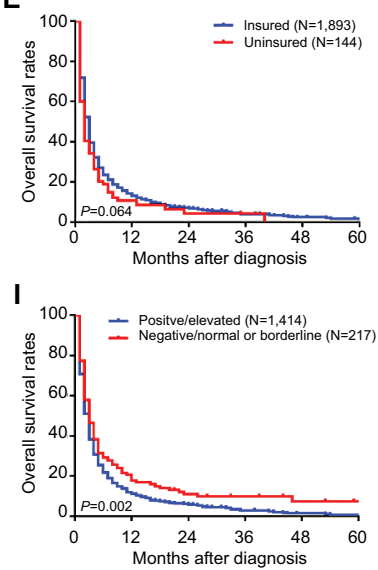

B

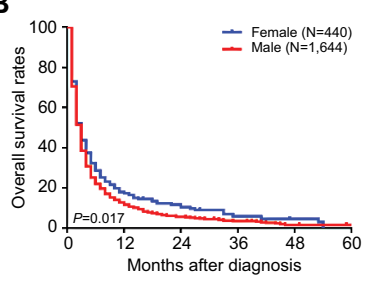

F
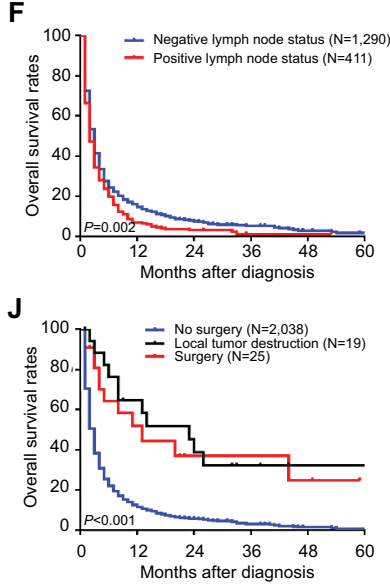

C

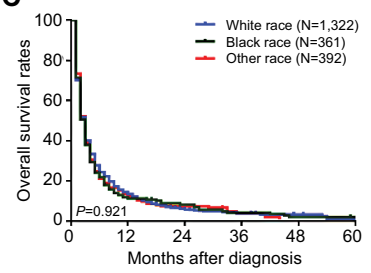

G

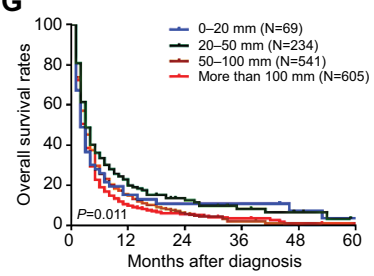

K

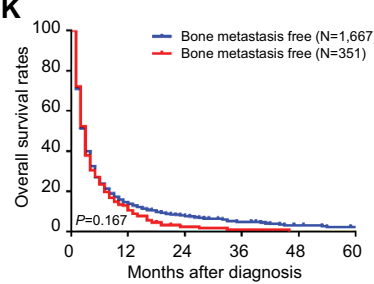

D

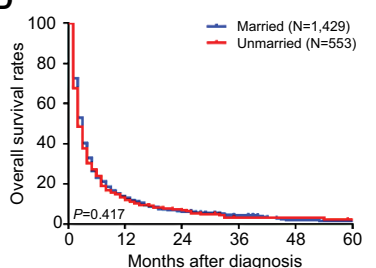

H
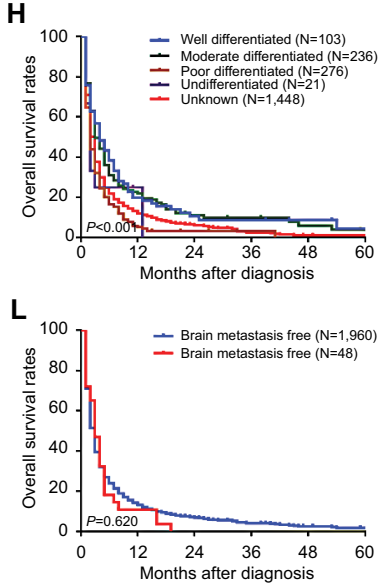

Figure 3 Kaplan-Meier analysis of overall survival in hepatocellular carcinoma patients with initial lung metastasis stratified by (A) age at diagnosis, (B) gender, (C) race, (D) marital status at diagnosis, (E) insurance status at diagnosis, (F) lymph node status, (G) maximum primary tumor size, (H) primary tumor differential grade, (I) alphafetoprotein level, $(\mathbf{J})$ surgery for primary tumor, $(\mathbf{K})$ bone metastasis, and $(\mathbf{L})$ brain metastasis.

A

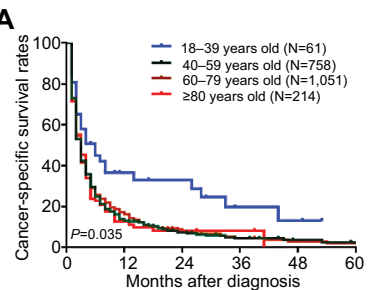

E
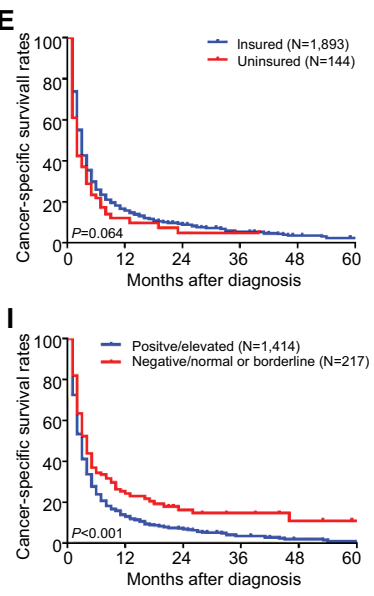

B

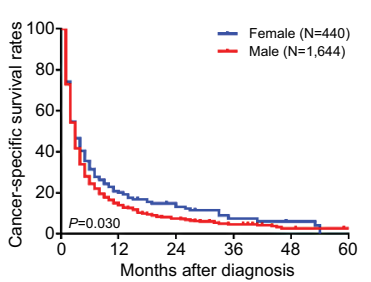

$\mathbf{F}$

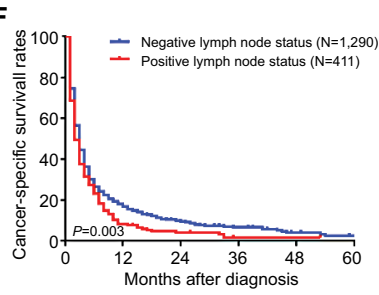

$\mathbf{J}$

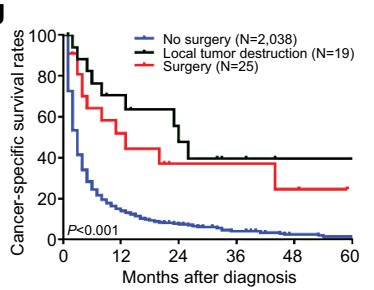

C

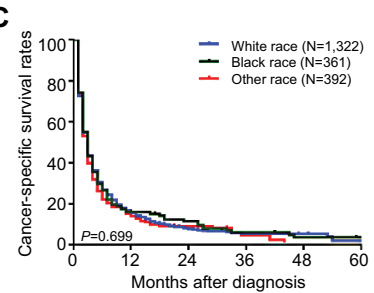

G

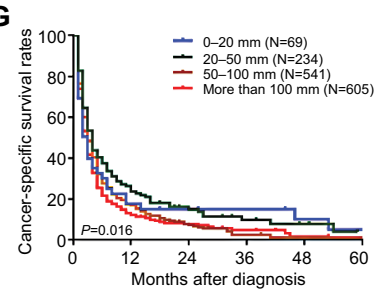

K

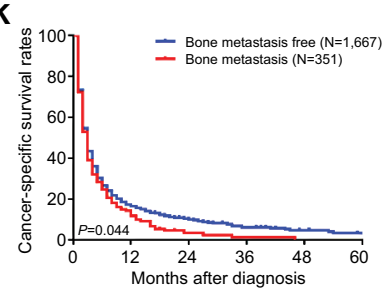

D

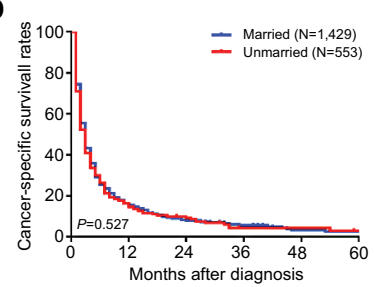

H
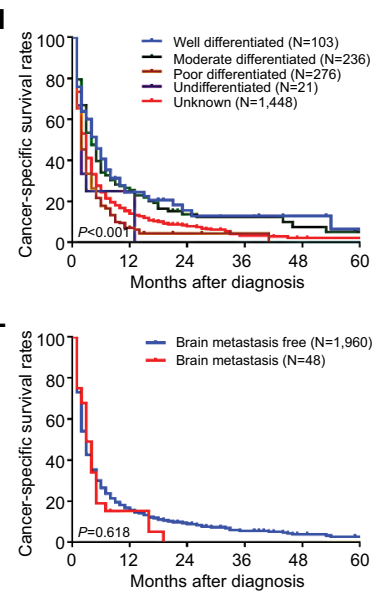

Figure 4 Kaplan-Meier analysis of cancer-specific survival in hepatocellular carcinoma patients with initial lung metastasis stratified by (A) age at diagnosis, (B) gender, (C) race, (D) marital status at diagnosis, (E) insurance status at diagnosis, (F) lymph node status, (G) maximum primary tumor size, (H) primary tumor differential grade, (I) alpha-fetoprotein level, $(\mathbf{J})$ surgery for primary tumor, $(\mathbf{K})$ bone metastasis, and $(\mathbf{L})$ brain metastasis. 
Table 2 Univariate and multivariate analysis of overall survival in patients with primary hepatocellular carcinoma lung metastasis in SEER database (2010-20I5)

\begin{tabular}{|c|c|c|c|c|}
\hline \multirow[t]{2}{*}{ Variables } & \multicolumn{2}{|l|}{ Univariate analysis } & \multicolumn{2}{|c|}{ Multivariate analysis } \\
\hline & HR (95\% CI) & $P$-value & HR (95\% Cl) & $P$-value \\
\hline \multicolumn{5}{|l|}{ Gender } \\
\hline Female & Reference & & & \\
\hline Male & $1.089(0.974-1.219)$ & 0.134 & & \\
\hline \multicolumn{5}{|l|}{ Age at diagnosis (years) } \\
\hline $18-39$ & Reference & & Reference & \\
\hline $40-59$ & $1.793(1.321-2.434)$ & $<0.001$ & $1.860(1.365-2.533)$ & $<0.001$ \\
\hline $60-79$ & $1.774(1.310-2.402)$ & $<0.001$ & $1.883(1.384-2.563)$ & $<0.001$ \\
\hline$\geq 80$ & $1.868(1.345-2.595)$ & $<0.001$ & $2.068(1.478-2.896)$ & $<0.001$ \\
\hline \multicolumn{5}{|l|}{ Race } \\
\hline White & Reference & & & \\
\hline Black & $0.975(0.864-1.100)$ & 0.680 & & \\
\hline Others & $0.942(0.836-1.06 \mathrm{I})$ & 0.322 & & \\
\hline Unknown & NA & NA & & \\
\hline \multicolumn{5}{|l|}{ Marital status at diagnosis } \\
\hline Married & Reference & & Reference & \\
\hline Unmarried & $\mathrm{I} .198(1.003-1.43 \mathrm{I})$ & 0.046 & $1.134(1.019-1.261)$ & 0.021 \\
\hline Unknown & NA & NA & NA & NA \\
\hline \multicolumn{5}{|l|}{ Insurance status } \\
\hline Insured & Reference & & & \\
\hline Uninsured & $1.088(0.982-1.206)$ & 0.107 & & \\
\hline Unknown & NA & NA & & \\
\hline \multicolumn{5}{|c|}{ Alpha-fetoprotein interpretation } \\
\hline Positive/elevated & Reference & & Reference & \\
\hline Negative/normal & $0.811(0.695-0.946)$ & 0.008 & $0.882(0.754-1.030)$ & 0.113 \\
\hline Borderline & $0.140(0.137-1.324)$ & 0.140 & $0.473(0.152-1.470)$ & 0.196 \\
\hline Unknown & NA & NA & NA & NA \\
\hline \multicolumn{5}{|l|}{ Surgery for primary site } \\
\hline No surgery & Reference & & Reference & \\
\hline Local tumor destruction & $0.268(0.15 \mathrm{I}-0.473)$ & $<0.001$ & $0.282(0.159-0.500)$ & $<0.001$ \\
\hline Surgery & $0.335(0.20 \mathrm{I}-0.557)$ & $<0.001$ & $0.390(0.232-0.654)$ & $<0.001$ \\
\hline Unknown & NA & NA & NA & NA \\
\hline \multicolumn{5}{|c|}{ Maximum primary tumor size $(\mathrm{mm})$} \\
\hline $0-20$ & Reference & & & \\
\hline $20-50$ & $0.923(0.696-1.225)$ & $0.58 \mathrm{I}$ & & \\
\hline $50-100$ & $1.059(0.8 \mid 4-1.379)$ & 0.669 & & \\
\hline$\geq 100$ & $1.182(0.909-1.536)$ & 0.212 & & \\
\hline Unknown & NA & NA & & \\
\hline \multicolumn{5}{|l|}{ Lymph node status } \\
\hline Negative & Reference & & Reference & \\
\hline Positive & $1.152(1.026-1.294)$ & 0.017 & $1.099(0.977-1.236)$ & 0.117 \\
\hline Unknown & NA & NA & $\mathrm{NA}$ & NA \\
\hline \multicolumn{5}{|l|}{ Bone metastasis } \\
\hline No & Reference & & & \\
\hline Yes & $1.010(0.896-1.138)$ & 0.876 & & \\
\hline Unknown & I.20I (0.928-I.555) & 0.165 & & \\
\hline \multicolumn{5}{|l|}{ Brain metastasis } \\
\hline No & Reference & & & \\
\hline Yes & $\mathrm{I} .073(0.798-\mathrm{I} .443)$ & 0.639 & & \\
\hline Unknown & NA & NA & & \\
\hline \multicolumn{5}{|l|}{ Grade } \\
\hline Well differentiated & Reference & & Reference & \\
\hline Moderately differentiated & $1.159(0.902-1.489)$ & 0.247 & I.206 (0.938-I.55I) & 0.145 \\
\hline Poorly differentiated & $1.705(1.337-2.176)$ & $<0.001$ & $1.732(1.355-2.213)$ & $<0.001$ \\
\hline Undifferentiated & $1.912(1.163-3.142)$ & 0.011 & $2.217(1.342-3.661)$ & 0.002 \\
\hline Unknown & NA & NA & NA & NA \\
\hline
\end{tabular}

Abbreviation: SEER, Surveillance, Epidemiology, and End Results. 
Table 3 Univariate and multivariate analysis of cancer-specific survival in patients with primary hepatocellular carcinoma lung metastasis in SEER database (2010-2015)

\begin{tabular}{|c|c|c|c|c|}
\hline \multirow[t]{2}{*}{ Variables } & \multicolumn{2}{|l|}{ Univariate analysis } & \multicolumn{2}{|c|}{ Multivariate analysis } \\
\hline & HR (95\% Cl) & $P$-value & HR (95\% Cl) & $P$-value \\
\hline \multicolumn{5}{|l|}{ Gender } \\
\hline Female & Reference & & & \\
\hline Male & $1.105(0.982-1.244)$ & 0.098 & & \\
\hline \multicolumn{5}{|l|}{ Age at diagnosis (years) } \\
\hline $18-39$ & Reference & & Reference & \\
\hline $40-59$ & $1.716(1.255-2.347)$ & 0.001 & 1.738 (I.268-2.382) & 0.001 \\
\hline $60-79$ & $1.685(1.235-2.299)$ & 0.001 & 1.747 (I.277-2.39I) & $<0.001$ \\
\hline$\geq 80$ & $1.704(1.214-2.392)$ & 0.002 & $1.832(1.300-2.583)$ & 0.001 \\
\hline \multicolumn{5}{|l|}{ Race } \\
\hline White & Reference & & & \\
\hline Black & $0.936(0.823-1.065)$ & 0.317 & & \\
\hline Others & $0.97 \mid(0.858-\mid .099)$ & 0.643 & & \\
\hline Unknown & NA & NA & & \\
\hline \multicolumn{5}{|l|}{ Marital status at diagnosis } \\
\hline Married & Reference & & & \\
\hline Unmarried & $1.070(0.96 \mathrm{I}-\mathrm{I} .193)$ & 0.218 & & \\
\hline Unknown & NA & NA & & \\
\hline \multicolumn{5}{|l|}{ Insurance status } \\
\hline Insured & Reference & & Reference & \\
\hline Uninsured & $1.214(1.008-1.462)$ & 0.041 & $1.163(0.963-1.405)$ & 0.117 \\
\hline Unknown & $\mathrm{NA}$ & NA & NA & NA \\
\hline \multicolumn{5}{|c|}{ Alpha-fetoprotein interpretation } \\
\hline Positive/elevated & Reference & & Reference & \\
\hline Negative/normal & $0.734(0.621-0.868)$ & $<0.001$ & $0.792(0.669-0.937)$ & 0.007 \\
\hline Borderline & $0.152(0.021-1.079)$ & 0.060 & $0.174(0.024-1.235)$ & 0.080 \\
\hline Unknown & NA & NA & NA & NA \\
\hline \multicolumn{5}{|l|}{ Surgery for primary site } \\
\hline No surgery & Reference & & Reference & \\
\hline Local tumor destruction & $0.246(0.132-0.459)$ & $<0.001$ & $0.276(0.148-0.516)$ & $<0.001$ \\
\hline Surgery & $0.370(0.222-0.617)$ & $<0.001$ & $0.430(0.256-0.722)$ & 0.001 \\
\hline Unknown & NA & NA & NA & NA \\
\hline \multicolumn{5}{|c|}{ Maximum primary tumor size $(\mathrm{mm})$} \\
\hline $0-20$ & Reference & & & \\
\hline $20-50$ & $0.906(0.675-1.218)$ & 0.514 & & \\
\hline $50-100$ & $\mathrm{I} .049(0.797-\mathrm{I} .38 \mathrm{I})$ & 0.733 & & \\
\hline$\geq 100$ & $1.165(0.886-1.531)$ & 0.274 & & \\
\hline Unknown & NA & NA & & \\
\hline \multicolumn{5}{|l|}{ Lymph node status } \\
\hline Negative & Reference & & Reference & \\
\hline Positive & $\mathrm{I} .177(\mathrm{I} .043-\mathrm{I} .328)$ & 0.008 & $1.112(0.983-1.257)$ & 0.091 \\
\hline Unknown & NA & NA & NA & NA \\
\hline \multicolumn{5}{|l|}{ Bone metastasis } \\
\hline No & Reference & & & \\
\hline Yes & $1.043(0.922-1.181)$ & $0.50 \mathrm{I}$ & & \\
\hline Unknown & NA & NA & & \\
\hline \multicolumn{5}{|l|}{ Brain metastasis } \\
\hline No & Reference & & & \\
\hline Yes & $1.105(0.813-1.501)$ & 0.524 & & \\
\hline Unknown & NA & NA & & \\
\hline \multicolumn{5}{|l|}{ Grade } \\
\hline Well differentiated & Reference & & Reference & \\
\hline Moderately differentiated & $1.159(0.902-1.489)$ & 0.274 & $\mathrm{I} .182(0.907-\mathrm{I} .542)$ & 0.216 \\
\hline Poorly differentiated & $1.765(1.366-2.280)$ & $<0.001$ & $1.762(\mathrm{I} .362-2.28 \mathrm{I})$ & $<0.001$ \\
\hline Undifferentiated & $1.920(1.136-3.246)$ & 0.015 & 2.139 (1.259-3.633) & 0.005 \\
\hline Unknown & NA & NA & NA & NA \\
\hline
\end{tabular}

Abbreviation: SEER, Surveillance, Epidemiology, and End Results. 
chronal bone or brain metastasis, tumor poor pathological differentiation, and absence of surgery. This result suggested that clinicians should consider the possibility of lung metastasis when visiting HCC patients with above characteristics. Therefore, routine chest radiograph or low-dose computed tomography of lung was considered for these patients. Regrettably, other reported risk factors, such as microvascular invasion and tumor encapsulation, could not be assessed owing to no record in SEER.

Survival estimates elucidated that age below 40 years, female, negative lymph node status, smaller tumor size, better tumor differentiation, normal AFP level, and surgery received may contribute to an optimistic survival. Prognostic factors for HCCLM were further investigated. The HCC patients with characteristics of age at diagnosis older than 40 years, unmarried status, tumor poor differentiation, and existence of surgery had a significantly higher risk of mortality. According to these recognized factors, clinicians could approximately assess the survival and prognosis of HCCLM patients.

Collectively, age at diagnosis, surgery for primary tumor, and tumor differential grade were crucial factors for both risk and prognosis prediction. Although HCC patients younger than 40 years had higher risk for lung metastasis, their OS and CSS appeared the best performance. The underlying causes perhaps were younger patients usually held more positive attitude toward treatment and had better physical status to tolerant various treatment modalities. In addition, the majority of patients did not receive surgery in this study, which most likely led to a lower survival rate compared with the reported data. ${ }^{11}$ Consistent with previous reports, larger tumor size and positive AFP level predicted high risk for lung tumor colony formation. ${ }^{4,12}$ Notably, concomitant brain or bone metastasis had no impact on OS among HCCLM patients.

\section{Limitations}

First, only patients diagnosed after 2010 are enrolled for analysis because metastatic sites had been recorded until 2010. Second, inevitable bias exists because of absent details of tumor pathology and patient performance status. Third, SEER database represents merely US population, and other countries with high incidence of HCC were unable to be enrolled for global analysis. Last, the research is retrospective and the result still needs prospective trials to confirm a precise conclusion.

\section{Conclusion}

Generally, the incidence of initial pulmonary metastasis in HCC patients is $6.28 \%$ and the 5-year OS is still poor. Some clinicopathological features, such as age at diagnosis, tumor differential grade, and surgery for primary tumor, are highly predictive of HCCLM and significantly affect patients' survival. To our knowledge, the study is the first attempt to explore the epidemiological characteristics and identify the associated risk or prognostic factors of HCCLM using a population-based cancer registry database. The conclusion could be useful for a precise and individualized therapeutic schedule.

\section{Acknowledgments}

This work was kindly sponsored by the Natural Science Foundation of China $(81773089,81802903)$ and China National Key Projects for Infectious Disease (2017ZX10203207). The authors also acknowledge the contribution of the SEER program in the establishment and maintenance of the database.

\section{Disclosure}

The authors report no conflicts of interests in this work.

\section{References}

1. Forner A, Reig M, Bruix J. Hepatocellular carcinoma. Lancet. 2018; 391(10127):1301-1314.

2. Siegel RL, Miller KD, Jemal A. Cancer statistics, 2018. CA Cancer J Clin. 2018;68(1):7-30.

3. El-Serag HB. Hepatocellular carcinoma. N Engl J Med. 2011;365(12): $1118-1127$.

4. Yokoo T, Patel AD, Lev-Cohain N, Singal AG, Yopp AC, Pedrosa I. Extrahepatic metastasis risk of hepatocellular carcinoma based on $\alpha$-fetoprotein and tumor staging parameters at cross-sectional imaging. Cancer Manag Res. 2017;9:503-511.

5. Li GC, Ye QH, Dong QZ, Ren N, Jia HL, Qin LX. TGF beta1 and related-Smads contribute to pulmonary metastasis of hepatocellular carcinoma in mice model. J Exp Clin Cancer Res. 2012;31:93.

6. Katyal S, Oliver JH, Peterson MS, Ferris JV, Carr BS, Baron RL. Extrahepatic metastases of hepatocellular carcinoma. Radiology. 2000;216(3):698-703.

7. Wu W, He X, Andayani D, et al. Pattern of distant extrahepatic metastases in primary liver cancer: a SEER based study. J Cancer. 2017;8(12): 2312-2318.

8. Kuo TM, Chang KM, Cheng TI, Kao KJ. Clinical factors predicting better survival outcome for pulmonary metastasectomy of hepatocellular carcinoma. Liver Cancer. 2017;6(4):297-306.

9. $\mathrm{Hu} \mathrm{Z,} \mathrm{Li} \mathrm{W,} \mathrm{Huang} \mathrm{P,} \mathrm{et} \mathrm{al.} \mathrm{Therapeutic} \mathrm{significance} \mathrm{and} \mathrm{indications}$ of pulmonary metastasectomy for hepatocellular carcinoma following liver resection. Int J Surg. 2017;48:23-31.

10. Takahashi Y, Ikeda N, Nakajima J, et al. Prognostic analysis of surgical resection for pulmonary metastasis from hepatocellular carcinoma. World J Surg. 2016;40(9):2178-2185.

11. Yang T, Lu JH, Lin C, et al. Concomitant lung metastasis in patients with advanced hepatocellular carcinoma. World J Gastroenterol. 2012;18(20):2533-2539.

12. Ishii T, Hatano E, Yasuchika K, Taura K, Seo S, Uemoto S. High risk of lung metastasis after resection of hepatocellular carcinoma more than $7 \mathrm{~cm}$ in diameter. Surg Today. 2014;44(10):1900-1905.

13. Zeng Y, Zhang X, Liu J, Zhong Y, Wang X. Correlated factors of pulmonary metastasis of hepatocellular carcinoma. Chin J Dig Surg. 2013;12(9):668-671.

14. Ikai I, Itai Y, Okita K, et al. Report of the 15th follow-up survey of primary liver cancer. Hepatol Res. 2004;28(1):21-29. 


\section{Publish your work in this journal}

Cancer Management and Research is an international, peer-reviewed open access journal focusing on cancer research and the optimal use of preventative and integrated treatment interventions to achieve improved outcomes, enhanced survival and quality of life for the cancer patient. The manuscript management system is completely online and includes a very quick and fair peer-review system, which is all easy to use. Visit http://www.dovepress.com/testimonials.php to read real quotes from published authors.

Submit your manuscript here: https://www.dovepress.com/cancer-management-and-research-journal 